\title{
ON THE COMETARY HYDROGEN COMA AND FAR UV EMISSION
}

H. U. Keller

\section{INTRODUCTION}

Comet Tago-Sato-Kosaka (1969 IX, hereafter TSK) was the first medium bright comet passing by in the era of ultraviolet satellites. The Orbiting Astronomical Observatory (OAO-2), observed in January 1970 the strong Lyman alpha signal at $1216 \mathrm{~A}$ which led to the first detection of cometary hydrogen. The peak signal in the photometer field-of-view (FOV) of $10^{\prime}$ diameter was about $70 \mathrm{kR}$ (Code et al 1970) The resonance scattering emission of hydrogen was optically thick in the central part of the coma. A rocket experiment of Jenkins and Wingert (1972) using an objective grating spectrograph confirmed the OAO-2 observations Later in Spring 1970 comet Bennett (1970 II) was observed in La by 0AO-2 and by two photometers onboard the Orbiting reophysical Observatory (OGO-5) (Bertaux and Blamont, 1970; Keller and Thomas, 1973). A single coservation of the short periodic comet Enke was also achieved (Bertaux et al, 1973). Comet Kohoutek (1973XII) triggered a variety of ultraviolet experiments using satellites, Copernicus (Bohlin et a1.: I. A. U.) and Skylab (Keller et al., 1975; Carruthers et al, 1974$)$, as well as rockets (Feldman et al , 1974; Opal et a1., 1974) and the spaceprobe Mariner 10 (Broadfoot et al., 1974) on its way to Mercury. 
For hydrogen coma interpretations this review will rely heavily on recently published data of comet Bennett. Many observations of comet Kohoutek are still being analyzed.

The cometary hydrogen observations are reviewed, and theoretical interpretations of the results are followed by a brief summary of UV observations other than $L \alpha$.

\section{La OBSERVATIONS}

A spectrometer with a FOV of $2^{\prime} \times 8^{\prime}$ onboard OAO-2 was used to construct a $\mathrm{L} \alpha$ isophote map of the central region of the hydrogen cloud of comet Bennett on April 16, 1970 (Code et a1., 1972) The roughness in the contours --extending out to $2 \mathrm{kR}$-- is partly explained by contamination of the L $\alpha$ signal by resonance oxygen emission at 1304 A (Lillie, 1974). The observed diameter was about $3 \times 10^{6} \mathrm{~km}$. The apparent heliocentric velocity of the comet forms an angle of $52^{\circ}$ with the antisolar direction (and not of more than $90^{\circ}$ as indicated in the map) A comparison with recent $\mathrm{L} \alpha$ isophote maps of comet Kohoutek (Opal et al, 1974) shows the OAO-2 isophotes appreciably more irregular The details are probably not physically relevant

The French OGO-5 observations, which yielded twelve maps (Bertaux et al , 1973), were made during a special spin-up (spin axis parallel to apparent sun-comet direction) 
in April 1970. These photometer observations showed the hydrogen coma extending out to $250 \mathrm{R}$ or a length (in antisolar direction) of $15 \times 10^{6} \mathrm{~km}$, several orders of magnitude larger than its visible counterpart: In these observations the influence of the $L \alpha$ radiative pressure force is dominant. The resolution is somewhat worse than the FOV diameter of $40^{\prime}$ would have permitted. Because the special spin-up motion of the satellite complicated the data reduction, the location of the cometary nucleus is not known

A different set of observations were achieved by the University of Colorado photometer onboard OGO-5 when the comet passed fortuitously through the FOV during the normal satellite operation in late March 1970 (Keller and Thomas, 1973): Four tracks across the cometary hydrogen cloud revealed that the $L \alpha$ intensity as far out as $30 \times 10^{6} \mathrm{~km}$ in antisolar direction still reached a value of about $70 \mathrm{R}$ above the sky background ( $4400 \mathrm{R})$ A relatively large FOV of about $3^{\circ}$ diameter increased the sensitivity of this instrument.

The Mariner 10 satellite made similar observations of comet Kohoutek in January 1974. The multichannel spectrometer of the Kitt Peak Observatory scanned the comet out to about $25 \times 10^{7} \mathrm{~km}$ in tail direction (Broadfoot et al, 1974). The data evaluation is still in progress. 
By far the best $L \alpha$ isophotes (Fig 1) resulted from an observation of comet Kohoutek by an electrographic camera flown on a rocket on January 8, 1974 (Opal et al, 1974) The resolution was about $2^{\prime}$ or $10^{5} \mathrm{~km}$; the comet's heliocentric distance was $0.43 \mathrm{a} . \mathrm{u} .$, and the influence of the $L \alpha$ radiation pressure was strong The innermost isophotes show optical thickness effects on the antisolar side not visible on the Bennett isophote maps. Thirteen maps were achieved by a similar camera onboard Skylab between 26 November 1973 and 2 February 1974 (Carruthers et a1, 1974) Some of the pictures are, unfortunately, degraded by several adverse technical circumstances. An interpretation is difficult because of the proximity of the comet to the sun and because the absorption of the geocorona must be considered on several occasions. A knowledge of the cometary emission line profile is necessary to make these corrections. Some observational progress was achieved improving the coarse determination of the line profile of TSK by Code et al (1970) who found a linewidth corresponding to a Doppler velocity $\mathrm{v}_{\mathrm{D}} \simeq 5 \mathrm{~km} \mathrm{~s}^{-1}$ The narrow geocoronal absorption line scanned across the cometary emission at the comet's perigeum.

The EUV spectrograph of the Naval Research Laboratory on Skylab received $L \alpha$ line profiles of the central optically thick parts of comet Kohoutek's hydrogen coma shortly after 


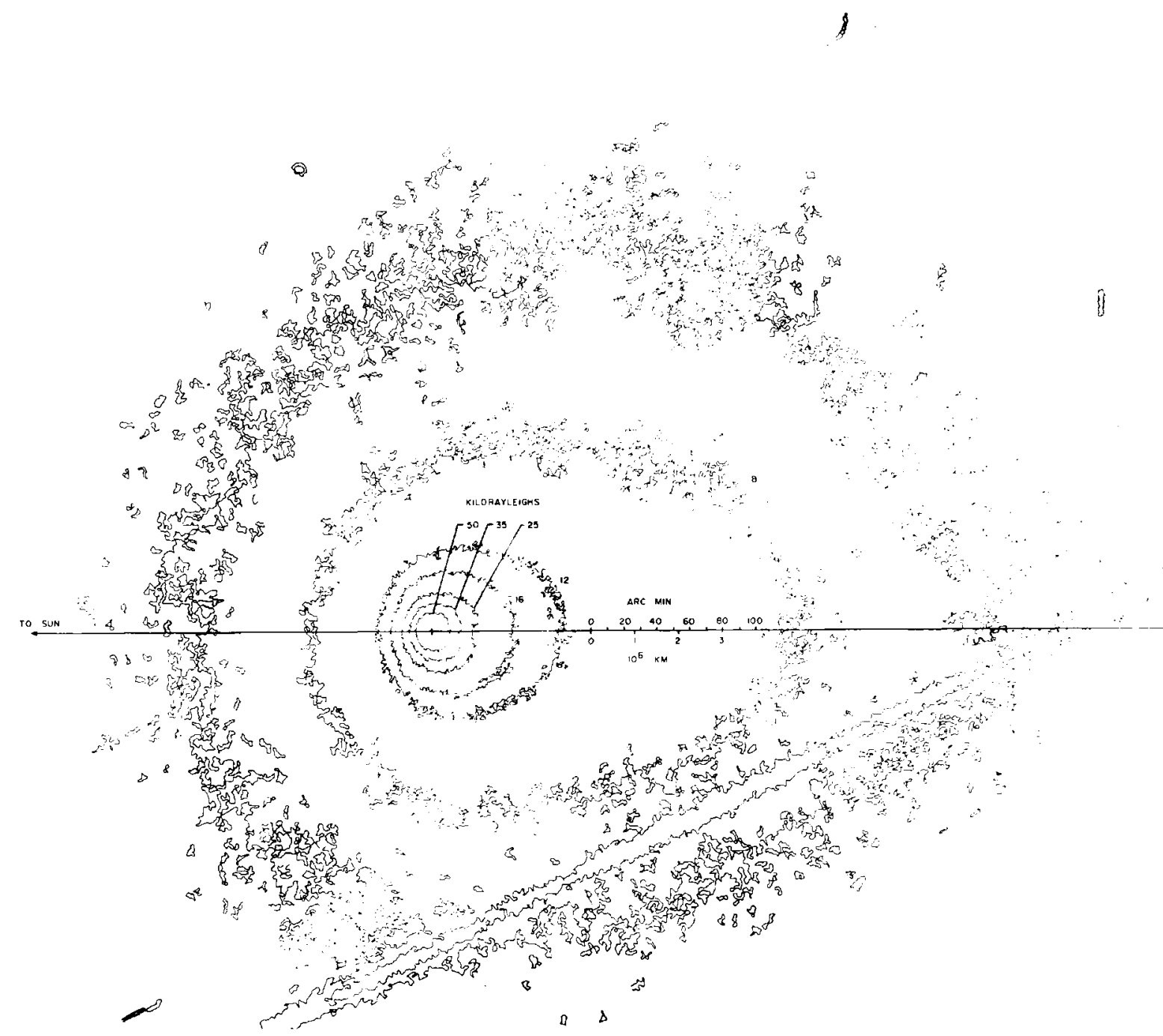

Figure 1 L $\alpha$ isophotes of comet Kohoutek, 81 January 1974, observed by Opal et al. (1974) The isophotes are represented by microdensitometer tracings converted to absolute emission rates ( $k R$ ) Linear and angular scales are indicated. Resolution in the inner parts is about $10^{5} \mathrm{~km}$ ( 2 arc $\left.\mathrm{min}\right)$ The outer isophotes in the lower part are deteriorated by a scratch on the film. 
perihelion. The observed broadened linewidth (FWHM $=014 \mathrm{~A}$ or $v_{D}=34 \mathrm{~km} \mathrm{~s}^{-1}$ ) agrees with the hydrogen outflow velocity $\mathrm{v}_{\mathrm{H}} \simeq 8 \mathrm{~km} \mathrm{~s}^{-1}$ if the small instrumental broadening and opacity effects are considered. The observations were not: good enough to deduce the actual cometary line profile (Keller et al, 1975) The Princeton University spectrometer onboard Copernicus (OAO-3) made observations in late January 1974, when the comet's heliocentric distance was about 1 a.u. (Bohlin et al., I. A. U.). The observed cometary Iinewidth corresponds to about $\mathrm{v}_{D}=9 \mathrm{~km} \mathrm{~s}-1$ since even the central region was predominantly optically thin.

High resolution observations with a Fabry-Perrot interferometer of comet Kohoutek detected the $\mathrm{H} \alpha$ emission of hydrogen. The determined linewidth corresponded to $\mathrm{v}_{\mathrm{D}}=8 \mathrm{~km} \mathrm{~s}^{-1}$ (Huppler et $\underline{\mathrm{al}}$, I A U.)

\section{INTERPRETATION}

Biermann (1968) described the overall features of the then still-hypothetical cometary hydrogen based on the dissociation of parent molecules like water and other hydrogen compounds. He called attention to the strong influence of the solar $L \alpha$ radiation pressure force and the limiting interaction with the solar wind. Keller (1971) 
pointed out that the photodissociation of hydrogen-containing parent molecules in many cases (including $\mathrm{H}_{2} \mathrm{O}$ ) yields high excess energies of more than $1 \mathrm{eV}$ These excess energies are almost completely transformed into kinetic energy of the liberated hydrogen atoms providing velocities of more than $10 \mathrm{~km} \mathrm{~s}^{-1}$. Depending on the overall gas production rate of a comet and, therefore, its heliocentric distance, $r$, these fast atoms are partially cooled by collisions. The inner region where collisions are possible has a radius on the order of $10^{4} \mathrm{~km}$, assuming a gas production rate on the order of $10^{30}$ molecule $\mathrm{s}^{-1}$ (typical for comet Bennett), and is therefore smaller than the region of dissociation which is about $10^{5} \mathrm{~km}$ at $\mathrm{r}=1$ a $\mathrm{u}$ (Keller, 1973a). The source of hydrogen is small if compared to the dimensions of the OGO-5 observations Keller (1971) suggested using Haser's (1966) fountain model -- originally developed for the visible coma -- for the interpretation of the hydrogen cloud This model assumes a point source at the cometary nucleus with radial outflow velocity distribution and includes the effects of solar radiation pressure and of a finite lifetime. The major drawback of the fountain mode1 is the neglect of the cometary motion during the lifetime of the hydrogen atoms (about 10 days at $r=1$ a $u$ ) Bertaux et al (1973) and Keller (1973b) used this model for the interpretation of the French OGO-5 data of comet Bennett 
The outflow velocity of the hydrogen atoms determines the extent of the sunward part of the isophotes (Fig 2); $\mathrm{v}_{\mathrm{H}}=8-9 \mathrm{~km} \mathrm{~s}^{-1}$ was found for the mean velocity of an assumed radial maxwellian velocity distribution The finite hydrogen lifetime, $t_{H}$, decreases the intensity of the isophotes the most on the down-sun side. $t_{H}$ was about $2 \times 10^{6} \mathrm{~s}$ (reduced to $r=1$ a.u.) in early April 1970. Bertaux et al (1973) found a decrease for later dates. This was attributed to an increase of the solar wind flux at solar latitudes higher than $45^{\circ}$ About $80 \%$ of the hydrogen atoms are ionized by charge exchange with solar wind protons; the rest are photoionized The hydrogen production rate, $\mathrm{Q}_{\mathrm{H}}$, -- the third free parameter of the fountain mode1 -- is determined from the absolute calibration An average value for $Q_{H}$ was $8 \times 10^{29} \mathrm{H}$ atom $\mathrm{s}^{-1}$ for $\mathrm{r}=0.8 \mathrm{a} . \mathrm{u}$.

Opal et al. (1974) also used the fountain model for a preliminary interpretation of their Kohoutek $\mathrm{L} \alpha$ isophote maps. The value for $v_{H}$ was confirmed.

The comparison of the computed isophotes with the observations of comet Bennett (Fig. 2) reveals some qualitative differences, particularly in antisolar direction (Keller 1973b). The tapering of the observed isophotes is not reproduced by the model The model shifts too much weight to the far down-sun parts, probably because of the incompleteness of the observations and the relatively 
Ly a Isophotes Comet Bennett (1970I) April I, 1970

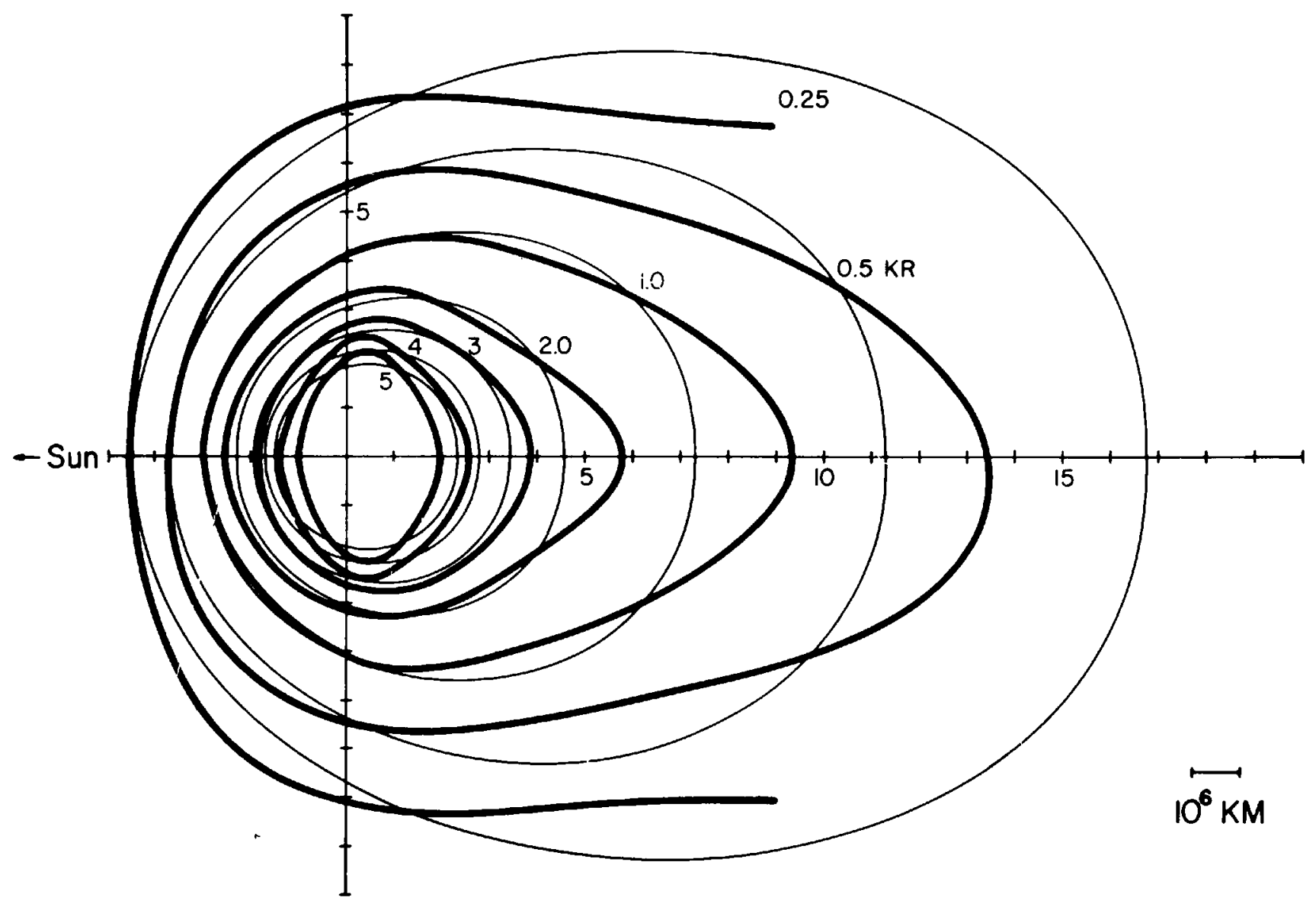

Figure 2 The observed La isophotes (Bertaux et a1, 1973), -, are compared to model calculations by Keller (1973b), - The model parameters are: $\mathrm{O}_{\mathrm{H}}=$ $1.2 \times 10^{30}$ atom $\mathrm{s}^{-1}, \mathrm{v}_{\mathrm{H}}=8 \mathrm{~km} \mathrm{~s}^{-1}, \mathrm{t}_{\mathrm{H}}=2.5$ $x 10^{6} \mathrm{~s}$, and a solar $\mathrm{L} \alpha$ center flux of $3.2 \mathrm{x}$ $10^{11} \mathrm{ph} \mathrm{s}^{-1} \mathrm{~cm}^{-2} \mathrm{~A}^{-2}$ is assumed. 
primitive model. The Kohoutek observations (Fig. 1) resemble the calculated isophotes better but do not match perfectly

Recently a more elaborate model was developed by Keller and Thomas (1975 and I A U ) This model uses methods simildr to the dust tail calculations. Hydrogen atoms leaving the nuclear region with zero ejection velocity form a curve in the orbital plane of the comet -- the syndyname -- at observation time The shape of the syndyname depends on the radiative pressure force and the cometary orbital elements. The points on the syndyname are interpreted as fictitious sources contributing to the line-ofsight density integral. This model accounts for the motion of the comet and the change of the hydrogen lifetime and production rate.

If the line-of-sight of the observations is nearly perpendicular on the orbital plane, the curvature of the hydrogen coma can be used to determine the solar L $\alpha$ flux. independent of any instrumental calibration Keller and Thomas achieved the following results from the University of Colorado photometer observations of comet Bennett shortly after perihelion in March 1970: $\mathrm{O}_{\mathrm{H}}=5.9( \pm 2) \times 10^{29}$ atom $\mathrm{s}^{-1}, t_{\mathrm{H}}=13(-0.3,+0.7) \times 10^{6} \mathrm{~s}$ both reduced to $r=1 \mathrm{a} \mathrm{u}$. These agree well with all the other Bennett results. The data at the outer boundaries of the hydrogen 
coma required a 50:50 mixture of two maxwellian velocity distributions, $v_{\mathrm{H}}=7$ and $21 \mathrm{~km} \mathrm{~s}^{-1}$, for a good fit The high velocity component is hard to detect on the antisolar side, it is masked by the low velocity $\mathrm{H}$ atoms The central solar $\mathrm{L} \alpha$ flux was determined to $5( \pm 1) \times 10^{11} \mathrm{ph} \mathrm{s}^{-1} \mathrm{~cm}^{-2} \mathrm{~A}^{-1}$ Keller (1973a) investigated the properties of the optically thick central parts of the cometary hydrogen coma The radiative transfer problem was solved for a purely radial outflow velocity distribution using Monte-Carlo techniques. Emission line profiles were determined for multiple and single scattering. This type of model calculation will have increasing importance for the interpretation of high resolution -- spatial and wavelength -observations. A comparison with the OAO-2 isophotes confirmed the interpretations of the fountain model for $Q_{H}$ and $\mathrm{v}_{\mathrm{H}} \cdot$

The physical parameters for particular observations of the hydrogen coma have been estahlished, and their variation with heliocentric distance must be investigated now The lifetime increases with $\mathrm{r}^{2}$ since the limiting effects of both solar wind and solar flux are diluted. However, the effect on the outflow velocity is not clear The French OGO-5 data of comet Bennett did not yield evidence for a systematic variation of the outflow velocity in the heliocentric distance interval from 061 to 1.0 a u A sys- 
tematic change in the hydrogen production rate was hardly detectable either, probably because the uncertainties of the observations were too large

OAO-2 photometer observations of comet Bennett in the heliocentric distance interval from 0.75 to 125 a.u. show a parallel decrease of the hydrogen and hydroxyl (OH) production rates with an r-exponent of $-23( \pm n 3)$ (Keller and Lillie, 1974; Lillie and Keller, I A. U. ). These results are unique because of the simultaneous $\mathrm{H}$ and $\mathrm{OH}$ observations with similar instruments and because they include the greatest heliocentric distance yet observed The parallel decrease and an $\mathrm{H} / \mathrm{OH}$ ratio of about 2 suggest a mutual parent molecule, probably water. The relatively small exponent 2.3 does not exclude more volatile molecules. No indication of a sudden drop of the vaporization of the parent molecule due to the re-radiation term in the equilibrium equation was found. These results allow conclusions on properties of the nucleus, e.g., its albedo (see Delsemme and Rud, 1973, and Keller and Lillie, 1974, for a more detailed discussion)

Delsemme (1973) had investigated similar, less complete OAO-2 data of comet TSK and found the variation of the production rates of the mutual parent moelcule of $\mathrm{H}$ and $\mathrm{OH}$ governed by the exponent -2.8 He concluded that only water evaporation can explain the data. 
Table I summarizes the results of the hydrogen observations of comet Bennett The preliminary results of comet Kohoutek observations do not show any significant differences. The hydrogen outflow velocities determined from L $\alpha$ isophotes (Opal et al, 1974) and line profile (Bohlin et al, I $A U$ ) seem to be equal.

The available hydrogen production rates of the comets TSK, Bennett, Kohoutek, and periodic comet Encke are illustrated in Fig. 3. The agreement for the Bennett observations are excellent. Undoubtedly, production rate determinations of cometary hydrogen based on the ultraviolet observations are by far more reliable than results for most other constituents

\section{TABLE I}

Comet Bennett (1970 II)

\section{Hydrogen}

Production Rate at 1 a $u$.

Lifetime at 1 a.u.

outflow velocity and component with

Production rate variation

for

$$
\begin{aligned}
& 0=54( \pm 2) \times 10^{29} \text { atom } \mathrm{s}^{-1} \\
& 1-25 \times 10^{6} \mathrm{~s} \\
& 7-9 \mathrm{~km} \mathrm{~s}^{-1} \\
& 220 \mathrm{~km} \mathrm{~s}^{-1} \\
& 0 \propto \mathrm{r}^{-n} 15<\mathrm{n}<26 \\
& 0.55<\mathrm{r}<125
\end{aligned}
$$




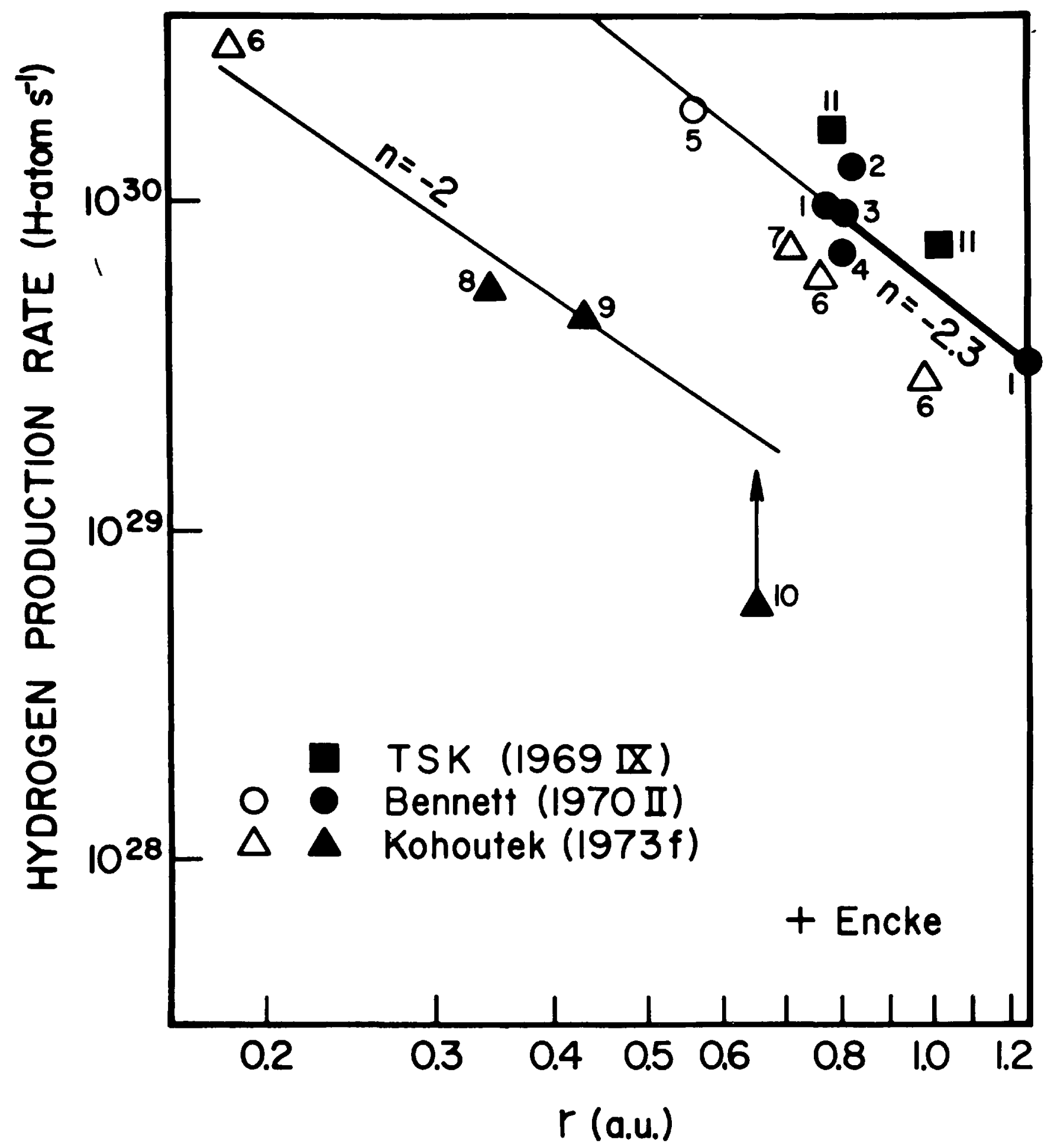

Figure 3 Hydrogen production rates of all observed comets: Open signs refer to the pre-perihelion, the filled ones to post-perihelion orbit. The ordinate is the heliocentric distance 
Figure 3 Legend

1. OAO-2, Keller and Lillie (1974) observations cover the interval between the edge points (heavier line). Observed slope, $\mathrm{n}=-2.3$.

2. OAO-2, Keller (1973a).

3. OGO-5, French Photometer, Bertaux et al (1973). A typical value is chosen. Observation interval from 0.6 to $1.0 \mathrm{a} . \mathrm{u}$

4. OGO-5, French Photometer, Keller (1973b). A typical value, representing the maximum deviation from average.

5. OGO-5, University of Colorado photometer, Keller and Thomas (1975)

6. Skylab, electrographic camera, Carruthers et al. (1974)

7. Ha observations, Huppler et al (I A U)

8. Spectrometer on rocket, Feldman et al (1974)

9. Electrographic camera on rocket, Opal et al. (1.974)

10. Mariner 10, UV spectrometer, Broadfoot et al. (1974).

11. OAO-2, Lillie (1974).

Comet Encke was observed by the French photometer on OGO-5, Bertaux et al (1973) 
Pre-perihelion comet Kohoutek's hydrogen production seemed to be slightly less than Bennett's; the L $\alpha$ and $H \alpha$ observations agree well. The hydrogen production rate was down by about a factor of five after perihelion as compared to comet Bennett. Thus, this decrease of the gas production (lessening the dust production too) explains comet Kohoutek's fainter visual brightness after perihelion. The hydrogen production peaked at perihelion and the exponent was approximately $-2\left(\mathrm{O}_{\mathrm{H}} \propto \mathrm{r}^{-2}\right)$. The final results of all Kohoutek observations (Skylab, Mariner 10, and Copernicus) will provide the hydrogen production rate variation from about 1 a.u to perihelion and back to the same heliocentric distance.

Also included in Fig. 3 are two points referring to comet TSK These are unpublished, preliminary results from OAO-2 observations. Surprisingly, the hydrogen production of comet TSK surpassed (about 50\%) that of comet Bennett whose intrinsic visual brightness was three magnitudes brighter. The superior visual brightness of the latter comet obviously stems from its tremendous dust production Comet Encke's hydrogen production rate was smaller by about two orders of magnitude

The questions remain how the hydrogen atoms are created and what their parent molecules are These large amounts of 
hydrogen atoms cannot be directly stored in the nucleus; the same holds for molecular hydrogen Clearly, the recently detected parent molecules, $\mathrm{HCN}, \mathrm{CH}_{3} \mathrm{CN}$, and $\mathrm{H}_{2} \mathrm{O}$ and other observed radicals contribute to the amount of atomic hydrogen. We shall not discuss in detail whether water $\left(\mathrm{H}_{2} \mathrm{O}\right)$ is dominating; only arguments directly connected with the ultraviolet observations will be mentioned. The strongest hint for the important role of water is based on the observations of the large $\mathrm{OH}$ production. The OAO-2 results for comet Bennett (Keller and Lillie, 1974) show that nearly all the hydrogen comes from water as assumed parent molecule of $\mathrm{OH}$, or at least one third in the extreme of the error limits. The Kohoutek observations yielded similar results Equally important, the $\mathrm{H}$ and $\mathrm{OH}$ production rates of comet Bennett decreased parallel between $\mathrm{r}=0.75$ and $1.25 \mathrm{a} u$ Probably, $\mathrm{OH}$ could not be synthesized efficiently enough by ion-molecule reactions in the inner coma (Oppenheimer, I A U.) and must be a dissociation product.

The conclusions from the values of the hydrogen outflow velocities observed and interpreted by several different methods are somewhat controversial. Table II shows the probable photodissociation processes connected with water Several laboratory experiments (e g, We1ge and Stuh1, 1967; 


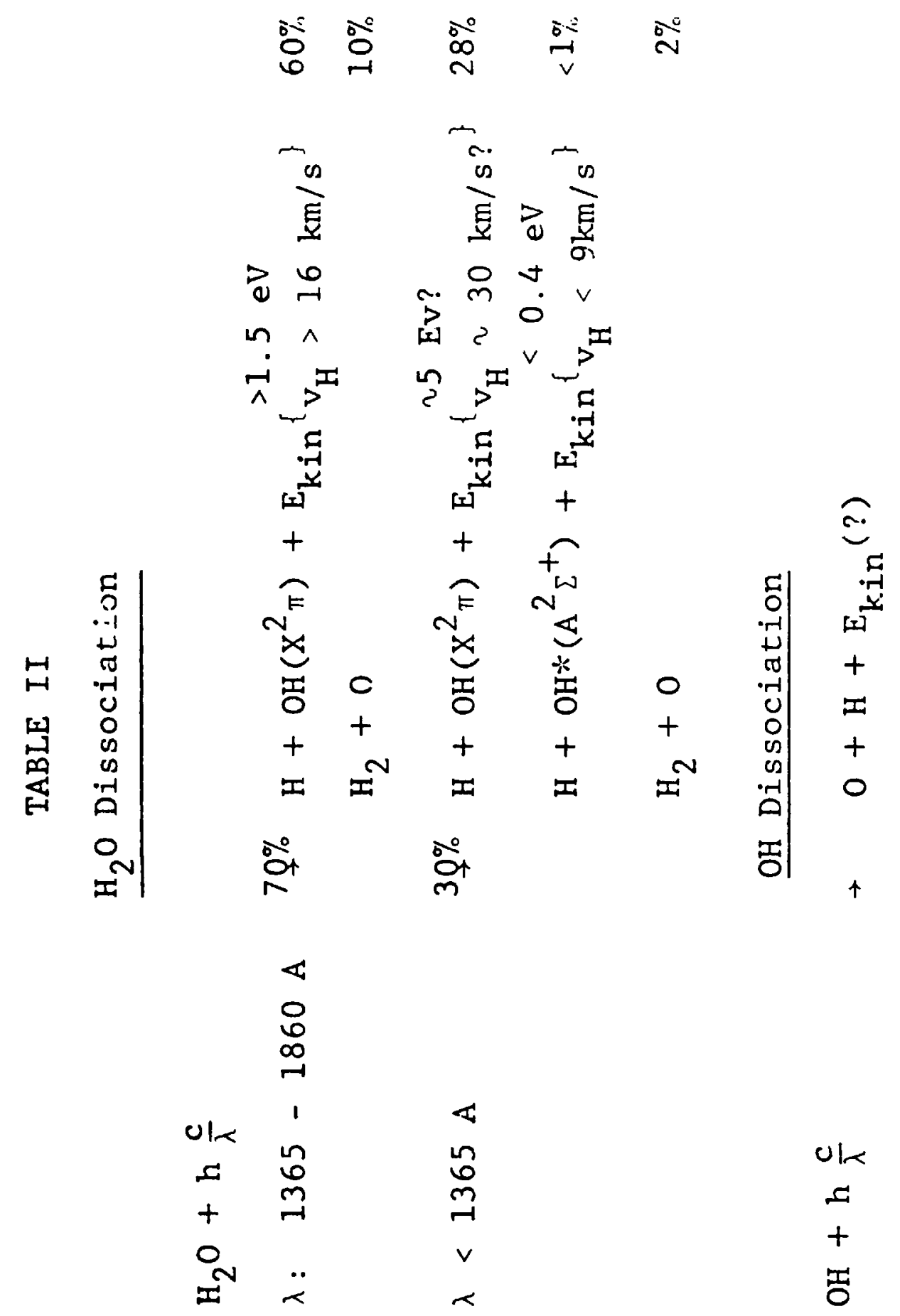


Carrington, 1964; and Stief, 1966) deal with the various dissociation branches of water; however, no measurements exist for $\mathrm{OH}$. The important $\mathrm{H}_{2} \mathrm{O}$ dissociations yield excess energies of more than $1.5 \mathrm{eV}$ which are nearly completely converted into translation energies of the $\mathrm{H}$ atoms The observed hydrogen velocity of $8 \mathrm{~km} \mathrm{~s}^{-1}$, however corresponds to only about $0.4 \mathrm{eV}$. Bertaux et al. (1973) estimated, based on a polytropic coma model of Mendis et al (1972), that only about $20 \%$ of the hydrogen atoms stemming from water dissociation are cooled, whereas the second generation $\mathrm{H}$ atoms from $\mathrm{OH}$ dissociation undergo practically no collisions. They concluded that the kinetic energy from this photodissociation must yield on $1 \mathrm{y}, 04 \mathrm{eV}$ or less. Most theoretical estimations, however, are $1 \mathrm{eV}$ or even more (Solomon, 1968). Wallis (1974) included the heating by dissociative excess energies in the inner coma more realistically, but his approximation also yielded only $30 \%$ thermalized hydrogen atoms

If thermalization determines the outflow velocity, $v_{H}$ should vary from comet to comet and with heliocentric distance. The sphere of collisions is proportional to the production rate and decreases in radius when the comet recedes. In addition, hydrogen atoms are created at a 
greater nuclear distance and live longer. An alternative is that all hydrogen atoms with $\mathrm{v}_{\mathrm{H}} \simeq 8 \mathrm{~km} \mathrm{~s}^{-1}$ are completely thermalized requiring a total gas production rate essentially higher than that observed of the $H$ atoms (Keller, $1971 ; 1973 a)$. The recent OH radio observations of comet Kohoutek (Biraud et al., 1974) seem to exclude collisional damping and result in rather large hydroxyl Doppler velocities of $3-4 \mathrm{~km} \mathrm{~s}^{-1}$

A small variation of the observed hydrogen outflow velocity cannot be excluded by the existing data but is not supported either A large portion of the hydrogen atoms may have high velocities (Keller and Thomas, I A. U) This high velocity component is difficult to observe More knowledge of the $\mathrm{H}_{2} \mathrm{O}$ and $\mathrm{OH}$ dissociation is badly needed to determine whether the observed hydrogen atom velocities are in agreement with the water photodissociation A stochastic treatment of the collision dominated inner coma region taking into account the partial relaxation of the initial non-thermal velocity distribution will make a detailed interpretation possible.

IV. NON-L $\alpha$ UV OBSERVATIONS

In the ultraviolet an identification of cometary constituents other than hydrogen is difficult. The emission rates are more than two orders of magnitude weaker than 
L $\alpha$ because of the low exciting solar flux and the smaller oscillator strengths of the transitions. The abundances are smaller too. Feldman et al (1974) made observations of comet Kohoutek on January 5, 1974, using two spectrometers covering the wavelength region 1200 to $3200 \mathrm{~A}$ (Table III) The figures are uncertain by at least a factor of two. The radial heliocentric velocity of the comet was large enough that the cometary atoms absorbed in the wings of the narrow solar emission lines (for $\lambda<1800$. A) introducing an additional uncertainty. This situation could be improved in future experiments by a series of observations at different cometary velocities (Feldman et al, I A. U).

Feldman et al (1974) concluded that their results are consistent with the assumption that water vapor dissociation is predominant The emission of carbon, CI, at 1657 and 1561 A was observed for the first time The fact that CI is only slightly less abundant $\left(Q_{0} / Q_{C} \simeq 3\right)$ than oxygen and hydroxyl is one of the most important results of all Kohoutek observations. Lillie (I. A. U.) confirmed the O:C ratio for comet Bennett from OAO-2 data Earlier, oxygen (1304 A) was found in about the same amount as hydrogen in comet Bennett (Code et al., 1972). Carbon is obviously by more than an order of magnitude more abundant than $\mathrm{CN}\left(\Omega_{\mathrm{H}}: \Omega_{\mathrm{CN}} \simeq 200\right.$ for comet Bennett, Code et al, 1972) It is probably not a minor constituent. Improved UV observations in future comets are important and might lead in addition to the 
TABLE III

UV Emissions of Comet Kohoutek (1973f)* 5 January $1974 \mathrm{r}=0.34 \mathrm{a} . \mathrm{u}$.

$\begin{array}{ccc}\text { Species } & \frac{\lambda[\mathrm{A}]}{\mathrm{O}\left[\mathrm{s}^{-1}\right]} \\ \mathrm{HI} & 1216 & 3.6 \times 10^{29} \\ \mathrm{OI} & 1304 & 1.4 \\ \mathrm{CI} & 1657 & 0.6 \\ \mathrm{CO} & 1510 & \leq 2.7 \\ \mathrm{H}_{2} & 1607 & \leq 0.3 \\ \mathrm{CO}_{2} & 2890 & \leq 101 \\ \mathrm{OH} & 3090 & 0.8\end{array}$

*Table from Feldman et a1 (1974)

Some values are revised

Species with UV emissions to be detected:

$\mathrm{He}, \mathrm{N}, \mathrm{N}_{2}, \mathrm{~N}_{2}^{+}, \mathrm{NO}, \mathrm{C}^{+}, \mathrm{CN}^{+}, \mathrm{O}^{+}, \mathrm{S}, \mathrm{Si}$, and metals 
detection of some of the constituents listed on the bottom of Table III

\section{OUTLOOK AND SUMMARY}

U1traviolet observations of more comets are badly needed to confirm our conclusions and to find which values are typical to all comets and which ones are specific to the individual comet or a group of comets. A new generation of UV instruments is available to make L $\alpha$ observations of comets down to about 10th visual magnitude We should try to duplicate the synoptic and simultaneous observations of the hydrogen and hydroxyl production rate variations of a comet and expand the heliocentric distance interval as far as possible in order to determine the sudden drop off point of the water evaporation. More difficult, but at least equally interesting, is the observation of the ratio of water dissociation products to the rest of the molecules and atoms and its variation with heliocentric distance. L $\alpha$ observations with good spatial resolution together with observations of the actual cometary emission profile will provide the information to determine the velocity distribution using models that include the relaxation of the hydrogen atoms.

In summary, the cometary $L \alpha$ observations have, without a shade of doubt, confirmed the relatively high overall gas production rates on the order of $10^{30}$ molecule $\mathrm{s}^{-1}$ of medium 
bright comets suggested by other observations and calculations in the last decade. Additional observations of oxygen and hydroxyl favor water to be one of the most abundant molecules in the coma at cometary heliocentric distances of about 1 a.u. and less. Water does not seem to outnumber other constituents by orders of magnitude in comet Kohoutek. The hydrogen production rate of comet Kohoutek was about a factor 5 less after perinelion and probably only slightly less pre-perihelion when compared to comet Bennett. The observed outflow velocities of the hydrogen atoms of both comets were about $7-10 \mathrm{~km} \mathrm{~s}^{-1}$, a value not yet understood If the high velocity component of $20 \mathrm{~km} \mathrm{~s}^{-1}$ or more comprises a larger amount, some of the quoted hydrogen production rates are actually higher. The intrinsic cometary brightness is only a very crude indicator of a comet's actual gas production rate as shown by the comparison of comets Bennett and TSK. The strength of the La emission favors these measurements as a standard procedure for observing future comets since they also provide the most accurate results on the total gas production rate and its variation with heliocentric distance.

Acknowledgements: This work was supported by a grant from the National Aeronautics and Space Administration, NCR 06-003-179. 


\section{REFERENCES}

Bertaux, J -L., Blamont, J. 1970, Compt. Rend. Acad. Sci 270; 1581 .

Bertaux, J.-L., Blamont, J., Festou, M. 1973, Astron. and Astrophys. 25, 415 .

Biermann, L 1968, JILA Report No 93.

Biraud, F., Burgois, G., Grovisier, J, Fillit, R., Gerard, E., Kazes, I. 1974, Astron. and Astrophys 34, 163

Bohlin, J D., Drake, J. F., Jenkins, E B, Keller, H U I.A.U.

Broadfoot, A. L., Belton, M. J. S , McElroy, M B , Kumar, S. 1974, Comet Kohoutek Workshop, Huntsville

Carrington, T 1964, J Chem. Phys. 41, 2012.

Carruthers, G R, Opal, C. B , Page, T. L, Meier, R R., Prinz, D. K. 1974, Icarus, in press

Code, A. D., Houck, T. E, Lillie, C F 1970, I A U Circ. 2201 .

Code, A. D., Houck, T E., Lillie, C F. 1972, The Scientific Results from the Orbiting Astronomical Observatory $(0 A O-2)$, Ed A D Code, NASA SP-310, 109

Delsemme, A. H 1973, Astrophys Letters 14, 163.

Delsemme, A H., Rud, D. A 1973, Astron and Astrophys $\underline{28}, 1$

Feldman, P D., Takacs, P Z, Fastie, W G, Donn, B 1974, Science 185,705 .

Feldman, P. D., Opal, C B., Meier, R R, Nicolas, K R, I. A. U

Haser, L. 1966, Congres Colloques L'Universite de Liege 37, 233.

Huppler, D. H Roessler, F L Scherb, F, Trauger, J T , I. A U.

Note: All references to I. A. U. are papers included in this volume. 
Jenkins, E. B., Wingert, D. W 1972, Astrophys I 174, 697.

Keller, H. U. 1971, Mitt. Astron Ges. 30, 143.

Keller, H. U. 1973a, Astron. and Astrophys 23, 269

Keller, H. U. 1973b, Astron and Astrophys 27, 51.

Keller, H U., Lillie, C. F. 1974, Astron and Astrophys 34, 187 .

Keller, H U., Thomas, G E 1973, Astrophys J L87

Keller, H U., Bohlin, J D, Tousey, R 1975, Astron and Astrophys. (in press)

Keller, H U., Thomas, G. E I A. U

Keller, H. U., Thomas, G E. 1975, Astron. and Astrophys., 39, 7.

Lillie, C. F 1974 private communication.

Lillie, C. F. I. A. U.

Lillie, C. F, Keller, H U, I. A. U

Mendis, D A, Holzer, T. E, Axford, W. I 1972, Astrophys Space Sci 15,313 .

Opal, C. B., Carruthers, Gr R., Prinz, D K., Meier, R. R. 1974, Science 185,702 .

Oppenheimer, M, I A. U.

Solomon, P M. 1968, Nature 217, 334.

Stief, L. J. 1966, J. Chem Phys 44, 277.

Wallis, M. K. 1974, Mon Not. R Astr Soc $\underline{166}, 181$

Welge, K. H, Stuhl, F 1967, J Chem. Phys 46, 2440.

NOTE: All references to I A $U$ are papers included in this volume 


\section{DISCUSSION}

D. J. Malaise: I have three comments on this review paper.

1. From what I have heard of stellar observations by OAO, the absolute calibration of the spectrometer and of the photometer was somewhat unreliable, and even relative calibration between the different experiments was not satisfactory. Here, moreover, you have to take into account the integration in the entrance aperture. What kind of uncertainty does that introduce in the relative production rates you have deduced?

2. I don't agree that these results are another proof that $\mathrm{H}_{2} \mathrm{O}$ is the main constituent of the comet. In fact, they seem to disprove it in two respects. First, the dissociation of $\mathrm{H}_{2} \mathrm{O}$ yields only the high velocity component of $\mathrm{H}(\mathrm{v}>$ $10 \mathrm{~km} / \mathrm{sec}$ ), so that about half the hydrogen observed in the coma (the low velocity component, $\mathrm{v} \sim 9 \mathrm{~km} / \mathrm{sec}$ ) needs another mother molecule than $\mathrm{H}_{2} \mathrm{O}$. Second, you showed that the variations of both $\mathrm{H}$ and $\mathrm{OH}$ with perihelion distance were remarkably parallel, while the production of $\mathrm{H}$ (the low velocity component for instance) through the dissociation of $\mathrm{OH}$ would give a $\mathrm{H} / \mathrm{OH}$ production rate greater at smaller perihelion distances.

3. You showed an observed profile for $\mathrm{H}$ and a 2 -component $(9 \mathrm{~km} / \mathrm{sec}+$ $21 \mathrm{~km} / \mathrm{sec}$ ) computed profile which was adjusted to fit the observations. I want to make a general comment about the fitting procedure in view of information theory. The two limits of the observed profile are essentially due to accidental errors, while there is a systematic deviation of the computed profile from the mean of the observed profiles. It is not relevant in the fitting procedure that the systematic deviation be kept within the accidental errors, because these two errors are of quite different nature.

As a matter of fact, if the correct physics were used to derive the theoretical profile, such large systematic deviations should not appear. If you allow such a misfit, a good mathematician will show you that there exists an infinite number of solutions to your problem. To choose one particular solution, you need to use additional constraints (other than your observed curve). These constraints can be simple hypothesis or personal views of how the model should be, etc. These constraints are used in parallel to the observations to define or select the fitting curve, but then it is an error of principle to claim that the observations prove the model. The model is an input and not an output of the fitting procedure; it can not be both.

\section{W. Jackson:}

1. The latest laboratory data for $\mathrm{H}_{2} \mathrm{O}$ is reviewed in my paper. However, it shows that in the first continuum $99 \%$ of the $\mathrm{H}_{2} \mathrm{O}$ dissociates into $\mathrm{H}+\mathrm{OH}$ $\left(\chi^{2} \pi\right)$, while in the second continuum $99 \%$ of the $\mathrm{if}_{2} \mathrm{O}$ dissociates into $\mathrm{H}+\mathrm{OH}$, 


\section{DISCUSSION (Continued)}

while $10 \%$ of the products are $\mathrm{H}_{2}+\mathrm{O}\left({ }^{1} \mathrm{D}\right)$. Further, the present data indicate that the excess energy is deposited into translational energy of the $\mathrm{H}$ product in both regions.

2. It seems unlikely that $\mathrm{CO}$ is the parent of $\mathrm{C}$ atoms, because laboratory data indicate that $\mathrm{CO}$ doesn't photodissociate in its first absorption band. This means that the lifetime against photodissociation is very long, since CO will only dissociate in a region below $1100 \AA$.

P. D. Feldman:

1. CO as a source of $\mathrm{C}$ is only an educated guess.

2. The new value for $Q_{O H}$ is $0.5 \times 10^{29} \mathrm{sec}^{-1}$ (Feldman, et al., paper 51). This gives $\mathrm{Q}_{\mathrm{OH}} / \mathrm{Q}_{\mathrm{H}} \sim 0.1$, which is probably outside experimental errors.

3. $\mathrm{CH}_{4} \rightarrow \mathrm{C}+4 \mathrm{H}$ would give a good fit to the data.

4. With a factor of 10 better sensitivity in UV observations, next time we should be able to see if $\mathrm{CO}$ is really present.

F. L. Whipple: It should be pointed out that the high production rates of some material like $\mathrm{H}_{2} \mathrm{O}$ was explicit in the early non-gravitational forces in comets. The necessary mass was unobserved at that time and had to have a vapor pressure something like $\mathrm{H}_{2} \mathrm{O}$ to confine the non-gravitational forces, as well as general comet activity, to the perihelion regions. The current evidence for many carbon compounds in comets relieves the need to postulate $\mathrm{CH}_{4}$, which seems to be incompatible with the presence of $\mathrm{CO}$ and $\mathrm{CO}_{2}$.

M. Festou: The velocity of the H-atoms is obtained by comparing fountainmodel and data, so we must use an experimental profile which is very dependent on this velocity, that is to say, the sunward profile. All observations fit with an $8 \mathrm{~km} / \mathrm{sec}$ component. If we use the anti-solar profile, we can see that the velocity is only a factor of proportionality, and the model fits the data when using a short lifetime for the $\mathrm{H}$-atoms; the velocity does not play any role. This is illustrated by the fact that we find both a short lifetime $\left(\sim 1.5 \times 10^{5} \mathrm{secs}\right)$ when using two different velocities $(20 \mathrm{~km} / \mathrm{sec}$ and $8 \mathrm{~km} / \mathrm{sec})$. Another way to fit the data when using a higher lifetime of the H-atoms is to take into account a complementary source in the anti-solar direction. I think the dissociation of $\mathrm{H}_{2} \mathrm{O}^{+}$can be this additional source, because $\mathrm{H}_{2} \mathrm{O}^{+}$is produced in a very small solid angle, which compensates its relatively low production rate. 